\title{
The new definitions of SEPSIS and SEPTIC SHOCK: What do they give us? An answer
}

\author{
Mervyn Singer \\ University College London, \\ Cruciform Building, \\ Gower Street, London WC1E 6BT, UK
}

Can I thank Dr Rodriguez and colleagues ${ }^{1}$ for engendering debate and discussion on the new Sepsis-3 definitions. ${ }^{2}$ This is a valuable exercise not only to raise queries and thoughts, but also to clarify misconceptions. Below, using a combination of science and data, I shall gently pick apart each of their assertions to demonstrate the flaws and inconsistencies in their arguments.

The main reason why SIRS was not included in the operationalization of the new sepsis definition was actually based on pathophysiology. The SIRS criteria are not particularly good in distinguishing a normal and appropriate host response to an infection from an inappropriate responseresulting in a more serious infection. A bad cold will thus qualify as 'sepsis' in the old terminology if accompanied, for example, by fever $>38{ }^{\circ} \mathrm{C}$ and a heart rate above $90 \mathrm{bpm}$. The new definition however describes a dysregulated, life-threatening host response that results in organ dysfunction. Whereas few patients die from a cold, despite having two or more SIRS criteria, a SOFA score $\geq 2$ related to the acute episode does indeed represent organ dysfunction and is associated with a $>10 \%$ risk of dying. ${ }^{2}$

The semantic argument posed by Rodriguez et al. of 7-in-8 patients admitted to ICU with infection-related organ failure having SIRS misses the point. Rather, the 1-in-8 who did not have the requisite SIRS criteria would not have qualified as having sepsis under the old definition despite having infection-related organ failure serious enough to require critical care, and to result in death in $16 \% .^{3}$ By comparison, the new criteria are necessarily allinclusive as these mandate new onset organ dysfunction.

The Bone paradigms have served a useful purpose but are now outdated. There was complete consensus among the Task Force, which specifically included many experts in 
sepsis pathophysiology, that sepsis represents much, much more than just an inflammatory (pro- and anti-) response. The failure of multiple immunomodulatory trials is testament to this fact. We surely need to take into account other pathways (metabolic, hormonal, bioenergetics, endothelial, etc.) responsible for producing organ dysfunction and not focus simply upon inflammation.

Dr Rodriguez and colleagues cite studies that relate the number of SIRS criteria to mortality risk. However, a rise in mortality from $7 \%$ to $17 \%$ for patients having 2 as opposed to the maximum 4 SIRS criteria does not match up to the difference in mortality ranging from $18 \%$ for 1 organ dysfunction, progressing stepwise to $68 \%$ for 5 organ dysfunctions. ${ }^{4}$ Furthermore, the mortality risk relating to the number of SIRS criteria in emergency room and ward patients is approximately three times lower than the equivalent SIRS score in ICU patients. ${ }^{5} \mathrm{~A}$ mortality risk predicated on organ dysfunction (using SOFA, LODS or qSOFA) is far more consistent, nothwithstanding the patient's hospital location. ${ }^{5}$

I am unaware of any hospital using SIRS criteria alone to trigger a Sepsis Code activation. If so, the poor ward response teams would be overwhelmed with multiple emergency referrals, running to see most hospital patients with a raised white count and a temperature! Many such patients are not infected, ${ }^{6}$ let alone need unnecessary antibiotics. With increasing concerns about antibiotic resistance and the need for good stewardship, we must be circumspect in throwing antibiotics around needlessly. Basing antibiotic prescriptions on SIRS criteria alone is thus worrisome. All major studies, e.g. the Surviving Sepsis Campaign registry ${ }^{7}$ and the Spanish multicentre before-after educational program study, ${ }^{8}$ have focused on patients admitted to critical care rather than general ward patients. Such patients already had organ dysfunction to merit ICU admission.

It is also important to stress that the sepsis definitions, and the criteria that describe them, are not intended to dictate clinical management. A sick, hypotensive yet fluid-resuscitated patient requiring vasopressors should not be treated any differently if his lactate is 2.1 (fulfilling the new septic shock criteria) rather than 1.9 (not 'septic 
shock'). Likewise, manifestations of organ dysfunction, e.g. hypotension, oliguria, dyspnoea, should obviously be actively treated without waiting for the clinician to perform a formal SOFA score and then proclaiming 'Eureka'.

The advantage of the SOFA score for operationalizing sepsis is that it utilizes simple physiological and biochemical tests that should be routinely performed in any sick patient where the clinician is concerned about organ dysfunction. However, the timing of SOFA scoring should be retrospective for coding, research and epidemiology purposes, and after the presumptive diagnosis of infection has been confirmed or refuted. We have offered a much more robust categorization than previously provided. This is sorely needed to improve upon a highly inconsistent epidemiology where sepsis and septic shock means different things to different people. This heterogeneity results in widely differing incidences and mortality rates. Such spurious differences impacts on epidemiology, quality improvement programs, and clinical trial design. ${ }^{9}$

I would also challenge the notion that international guideline campaigns implemented around the previous definitions have "dramatically decreased" mortality. These claims usually rely either on complicated statistical manipulations or huge increases in the denominator with a corresponding dilutional effect --- the Will Rogers Phenomenon. For example, one US study reported a fall in severe sepsis mortality from $40 \%$ to $27 \%$ between 2000 and $2007 .{ }^{10}$ Yet, in the same period the number of cases of severe sepsis rose massively from 300,270 to 781,725 , and the actual number of total deaths nearly doubled. A similar national ICD-10 database study has recently been published from Germany; ${ }^{11}$ in seven years (from 2007 to 2013) mortality from severe sepsis and septic shock fell from $49.5 \%$ to $43.6 \%$ yet the number of cases tripled and total deaths also doubled. Hardly the claimed "dramatic decrease" Dr Rodriguez and colleagues attest! Correcting for illness severity, there has been some improvement in mortality over time, as shown by recent Australasian3 and UK national data, ${ }^{4}$ yet this improvement is less marked for sepsis than for patients with non-septic critical illness (Shankar-Hari M., personal communication).

With respect to quickSOFA can I respectfully suggest the authors read the detail provided in both the main definitions paper ${ }^{2}$ and the accompanying paper by Seymour et al. ${ }^{5}$ Twenty- 
eight commonly collected physiological and biochemical variables (including the SIRS criteria and lactate) were evaluated; regression analysis identified the three qSOFA criteria as the best determinants of mortality risk, and a respiratory rate cut-off of 22 breaths/min was superior to the SIRS respiratory rate cut-off of 20. Unlike the totally arbitrary selection by Bone et al. of the SIRS cut-offs, ${ }^{12}$ qSOFA was developed from data collected on hundreds of thousands of patients. They also misunderstand the point of qSOFA: we wrote that "adult patients with suspected infection can be rapidly identified (with qSOFA) as being more likely to have poor outcomes typical of sepsis. ${ }^{2 \prime}$ We were also at pains to stress in the paper that qSOFA is not part of the new definition of sepsis, as re-emphasized in a recent editorial, ${ }^{13}$ but "be used to prompt clinicians to further investigate for organ dysfunction, to initiate or escalate therapy as appropriate, and to consider referral to critical care or increase the frequency of monitoring, if such actions have not already been undertaken. The task force considered that positive qSOFA criteria should also prompt consideration of possible infection in patients not previously recognized as infected." ${ }^{2}$ We also wrote "It is crucial, however, that failure to meet 2 or more qSOFA or SOFA criteria should not lead to a deferral of investigation or treatment of infection or to a delay in any other aspect of care deemed necessary by the practitioners". ${ }^{2}$ The beauty of qSOFA --- acknowledging that it does need prospective validation in different healthcare settings --- is that it can be performed by any healthcare practitioner at the bedside in just 1---2 $\mathrm{min}$--- unlike SIRS testing that requires an intrinsic delay of even several hours while blood tests are performed to measure white count and $\mathrm{PaCO} 2$.

I would also remind Dr Rodriguez and colleagues that all the studies claiming benefit from "essential therapeutic measures such as early administration of antibiotics" were all performed on patients with existing organ dysfunction, if not full-blown shock. The 'essential' nature of this particular argument is also challenged and undermined by a recent metaanalysis ${ }^{14}$ and even more recent prospective studies contesting this particular dogma [e.g. ${ }^{15,16}$. What is truth, indeed?!

Finally, they question the new clinical characterization of septic shock. I would sincerely hope that hyperlactataemia persisting after initial resuscitation does not define a terminal event, as Rodriguez and colleagues suggest. From the Surviving Sepsis Campaign registry of 
28,150 patients admitted to ICUs with infection-related organ failure and adequate fluidresuscitation, upon which we based our criteria, mortality was $42.3 \%$ in patients having both hypotension (MAP $<65 \mathrm{mmHg}$ ) and persisting hyperlactataemia ( $>2 \mathrm{mmol} / \mathrm{l}) .{ }^{17}$ Mortality was considerably lower in patients meeting only one or none of these criteria, i.e. $25.7 \%$ with hyperlactataemia alone, $30.1 \%$ with fluid-resistant hypotension alone, and $25 \%$ with organ dysfunction despite a normal lactate and blood pressure. The 'unexpected low mortality' they note in recent clinical septic shock trials is actually not unexpected when the criteria used to define shock in these trials are more closely examined. Take for instance, the Early Goal-Directed Therapy studies where a lactate $>4$ irrespective of fluid resuscitation, or refractory hypotension (systolic $\mathrm{BP}<90 \mathrm{~mm} \mathrm{Hg}$ or MAP $<65 \mathrm{~mm} \mathrm{Hg}$ despite resuscitation with at least 1 I of intravenous fluid) determined study inclusion. ${ }^{18-20}$ 'Shock' in many of these patients was rapidly reversed with fluid alone and did not need vasopressors, mechanical ventilation or renal replacement therapy. However, only $15---20 \%$ of patients entered into these trials fulfilled the much tighter clinical criteria required by the new septic shock definition. Our systematic review showed multiple criteria have been used in the literature to describe septic shock. ${ }^{17}$ This however generated a ten-fold variation in incidence and a four-fold variation in mortality. We thus need to talk the same language to make sensible national, international or temporal comparisons.

\section{Ethical disclosures}

Protection of human and animal subjects.

The authors declare that no experiments were performed on humans or animals for this study.

\section{Confidentiality of data.}

The authors declare that no patient data appear in this article.

Right to privacy and informed consent.

The authors declare that no patient data appear in this article.

\section{Funding}

None declared. 
Conflicts of interest

None declared.

\section{Acknowledgements}

None declared.

\section{References}

1. Rodríguez A, Martín-Loeches I, Yébenes JC. New definition of SEPSIS and SEPTIC SHOCK: what gives us? MedIntensiva. 2016 (in press).

2. Singer M, Deutschman CS, Seymour CW, Shankar-Hari M, Annane D, Bauer M, et al. The third international consensus definitions for Sepsis and Septic Shock (Sepsis-3). J Am Med Assoc. 2016;315:801-10.

3. Kaukonen KM, Bailey M, Suzuki S, Pilcher D, Bellomo R. Mortality related to severe sepsis and septic shock among critically ill patients in Australia and New Zealand, 2000-2012. J Am Med Assoc. 2014;311:1308---16.

4. Shankar-Hari M, Harrison DA, Rowan KM. Differences in impact of definitional elements on mortality precludes international comparisons of sepsis epidemiology-a cohort study illustrating the need for standardized reporting. Crit Care Med. 2016 (in press).

5. Seymour CW, Liu VX, Iwashyna TJ, Brunkhorst FM, Rea TD, Scherag A, et al. Assessment of clinical criteria for sepsis: for the third international consensus definitions for Sepsis and Septic Shock (Sepsis-3). J Am Med Assoc. 2016;315:762-74.

6. Churpek MM, Zadravecz FJ, Winslow C, Howell MD, Edelson DP. Incidence and prognostic value of the systemic inflammatory response syndrome and organ dysfunctions in ward patients. Am J Respir Crit Care Med. 2015;192:958-64.

7. Rhodes A, Phillips G, Beale R, Cecconi M, Chiche J-D, De Backer D, et al. The surviving sepsis campaign bundles and outcome: results from the International Multicentre Prevalence Study on Sepsis (the IMPreSS study). Intensive Care Med. 2015;41: 1620-8. 8. Ferrer R, Artigas A, Levy MM, Blanco J, González-Díaz G, Garnacho-Montero J, et al. Improvement in process of care and outcome after a multicenter severe sepsis educational program in Spain. J Am Med Assoc. 2008;299:2294-303. 
9. Rhee C, Kadri SS, Danner RL, Suffredini AF, Massaro AF, Kitch BT, et al. Diagnosing sepsis is subjective and highly variable: a survey of intensivists using case vignettes. Crit Care. 2016; 20:89.

10. Kumar G, Kumar N, Taneja A, Kaleekal T, Tarima S, McGinley E, et al. Nationwide trends of severe sepsis in the 21st century (2000-2007). Chest. 2011; 140:1223-31.

11. Fleischmann C, Thomas-Rueddel DO, Hartmann M, Hartog CS, Welte T, Heublein S, et al. Hospital incidence and mortality rates of sepsis. Dtsch Arztebl Int. 2016;113:159-66.

12. Bone RC, Balk RA, Cerra FB, Dellinger RP, Fein AM, Knaus WA, et al. Definitions for sepsis and organ failure and guidelines for the use of innovative therapies in sepsis. The ACCP/SCCM Consensus Conference Committee. Chest. 1992;101:1644-55.

13. Vincent J-L, Martin GS, Levy MM. qSOFA does not replace SIRS in the definition of sepsis. Crit Care. 2016;20:210.

14. Sterling SA, Miller WR, Pryor J, Puskarich MA, Jones AE. The impact of timing of antibiotics on outcomes in severe sepsis and septic shock: a systematic review and metaanalysis. Crit Care Med. 2015;43:1907-15.

15. de Groot B, Ansems A, Gerling DH, Rijpsma D, van Amstel P, Linzel D, et al. The association between time to antibiotics and relevant clinical outcomes in emergency department patients with various stages of sepsis: a prospective multi-center study. Crit Care. 2015;19:194.

16. Fitzpatrick JM, Biswas JS, Edgeworth JD, Islam J, Jenkins N, Judge R, et al. Gram-negative bacteraemia; a multicentre prospective evaluation of empiric antibiotic therapy and outcome in English acute hospitals. Clin Microbiol Infect. 2016;22:244-51.

17. Shankar-Hari M, Phillips GS, Levy ML, Seymour CW, Liu VX, Deutschman CS, et al. Developing a new definition and assessing new clinical criteria for septic shock: for the third international consensus definitions for sepsis and septic shock (Sepsis-3). J Am Med Assoc. 2016;315:775-87.

18. The ProCESS Investigators. A randomized trial of protocol-based care for early septic shock. N Engl J Med. 2014;370:1683-93.

19. Peake SL, Delaney A, Bailey M, Bellomo R, ARISE Investigators, ANZICS Clinical Trials Group, et al. Goal-directed resuscitation for patients with early septic shock. N Engl J Med. 2014;371:1496-506. 
20. Mouncey PR, Osborn TM, Power GS, Harrison DA, Sadique MZ, Grieve RD, et al. Trial of early, goal-directed resuscitation for septic shock. N Engl J Med. 2015;372:1301-11. 